\title{
Glinical Analysis of Ten Cases with Surgically Treated Brain Stem Cavernous Angiomas
}

\author{
Kazuo Mizoi, Takashi Yoshimoto* and Jiro Suzuki* \\ Depeartment of Neurosurgery, Kohnan Hospital, Sendai \\ 982, and *Division of Neurosurgery, Tohoku University \\ School of Medicine, Sendai 980
}

\begin{abstract}
Mizor, K., Yoshimoto, T. and Suzuni, J. Clinical Analysis of Ten Cases with Surgically Treated Brain Stem Cavernous Angiomas. Tohoku J. Exp. Med., 1992, 166 (2), 259-267 — Ten cases of surgically treated brain stem cavernous angiomas are reported in this paper, and their clinical and radiological features are reported. There were 9 males and 1 female with ages ranging from 9 to 69 years (average 36 years). Four of the cavernous angiomas were located in the dorsal pons, two in the middle cerebellar peduncle, two in the hypothalamus, and one each in the cerebral peduncle and the superior colliculus. All the ten patients came to medical attention because of the sudden onset of neurological abnormalities following brain stem hemorrhage. Six of the 10 patients showed neurological deterioration due to rebleeding at various intervals ranging from 10 days to 2 years. After intravenous injection of iodinated contrast materials, negative contrast enhancement was commonly seen. In a few cases, there was faint enhancement of the nodule at the periphery of the hematoma. Serial computed tomography (CT) scans over a period revealed an unchanged hyperdense mass lesion, which was thought to be a most characteristic and indicative CT feature of the cavernous angioma. On magnetic rersonance imaging (MRI), the combination of a mixed signal intensity with a surrounding rim of decreased signal intensity (hemosiderin rim) suggested the presence of a cavernous angioma. Enhanced MRI, using gadopentate dimeglumine, was thought to be more sensitive than contrast enhanced CT in detecting cavernous angiomas. All these cases underwent surgery with good results. Abnormal vascular tissue in the wall of the hematoma cavity was recognized at operation and pathological confirmation was obtained in all cases. These data indicate that brain stem cavernous angiomas can be surgically removed with acceptable morbidity. —— cavernous angioma; brain stem; MRI ; CT scan
\end{abstract}

Since the introduction of computed tomography (CT) and magnetic resonance imaging (MRI), considerable attention has been given to cavernous angiomas (or angiographically occult arteriovenous malformations) which are the cause of bleeding in the brain stem. Although there have been few previous reports concerning the surgical excision of such lesion (Veerapen et al. 1986 ; Yoshimoto

Received December 27, 1991 ; revision accepted for publication January 25, 1992.

Reprint requests : Kazuo Mizoi, M.D., Department of Neurosurgery, Kohnan Hospital, 4-20-1 Nagamachi-minami, Taihaku-ku, Sendai 982, Japan. 
and Suzuki 1986 ; Ondra et al. 1988 ; Konovalov et al. 1990 ; Tung et al. 1990 ; LeDoux et al. 1991), we have experienced ten such cases of direct surgery and report them here with special emphasis on the characteristic features in computerized imaging studies and the determination of the indications for direct surgery.

\section{Clinical Materials and Results}

During the ten year period between 1980 and 1990, we experienced ten cases of cavernous angiomas of the brain stem, for wihch direct surgery was undertaken (Table 1). Ages ranged between 9 and 69 years (mean of 36 years), which a slight prevalence for the fourth decade (four patienst in their 30s). The vast majority were male (nine cases), with only one female case. A wide variety of symptoms were seen at onset, depending upon the site of the hemorrhage, but none showed severe disturbance of consciousness. Following the initial attack, deterioration due to rebleeding was frequently seen, and confirmed in CT scans in six of the ten cases. Rebleeding occurred at any time from 10 days to 2 years after the first attack, and there was no predominant period for rebleeding.

The site of the angiomas and hematomas were in the dorsal pons (4 cases), brachium pontis ( 2 cases), hypothalamus ( 2 cases), superior colliculus ( 1 cases) or extending from the midbrain to the pons ( 1 cases). The dorsal pons was clearly the favored site for brain stem cavernous angiomas.

The presence of the nonspecific intra-axial hematoma was the main CT finding in these cases, but the characteristic feature was the prolonged existence of hyperdense lesion on noncontrast CT long after the high density due to a hematoma would usually have disappeared. Although there were individual differences regarding contrast enhanced CT, in general the enhancement effect was mild.

MRI was performed in five of the ten cases. Characteristic findings were:

TABLE 1. Summary of cases

\begin{tabular}{|c|c|c|c|c|c|c|c|}
\hline Case & $\begin{array}{c}\text { Age } \\
\text { (years) }\end{array}$ & Sex & Symptoms & Location & $\begin{array}{l}\text { Rebleeding } \\
\text { (intervals) }\end{array}$ & Operation & Outocme \\
\hline 1 & 35 & M & Cerebellar signs & Pons & $+(3$ months $)$ & 1980 & Good \\
\hline 2 & 40 & M & Hemianopsia & Hypothalamus & $+(6$ months $)$ & 1982 & Good \\
\hline 3 & 16 & $\mathrm{M}$ & Hemiparesis & Pons & - & 1984 & Good \\
\hline 4 & 69 & M & Cerebellar signs & Brach. conj. & $+(14$ days $)$ & 1984 & Good \\
\hline 5 & 9 & $\mathrm{~F}$ & Cerebellar signs & Brach. conj. & - & 1985 & Good \\
\hline 6 & 39 & M & MLF synd. & Pons & $+(10$ days $)$ & 1986 & Good \\
\hline 7 & 20 & M & Hemiparesis & Cerebral ped. & - & 1986 & Good \\
\hline 8 & 36 & M & Millard-Gublar & Pons & $+(44$ days $)$ & 1988 & Good \\
\hline 9 & 39 & M & Upward gaze palsy & Sup. colliculus & $+(2$ years $)$ & 1989 & Good \\
\hline 10 & 60 & M & Dementia & Hypothalamus & - & 1990 & Good \\
\hline
\end{tabular}




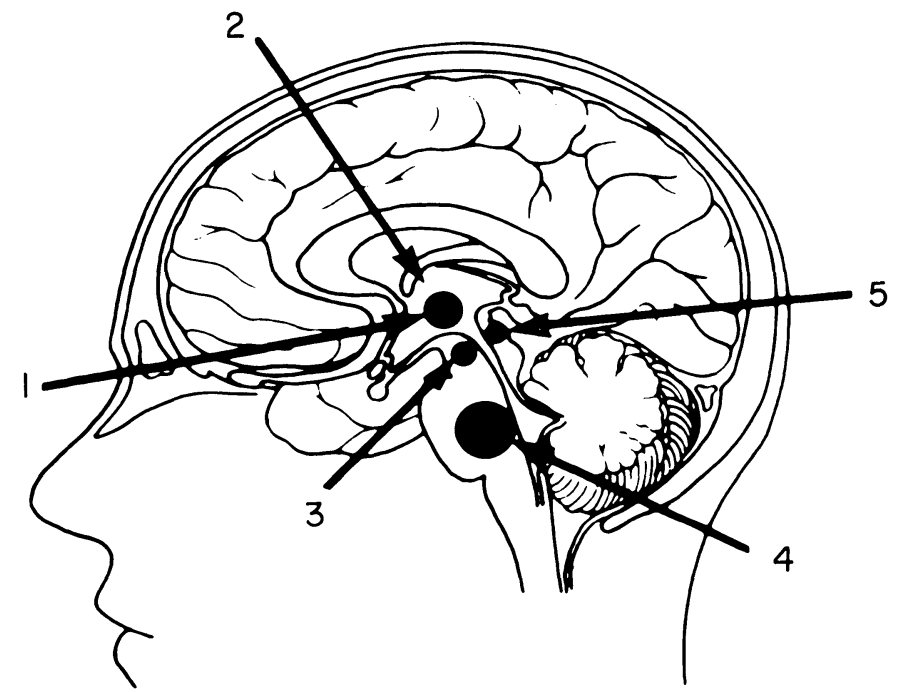

Fig. 1. Schematic representation of the various approaches to the brain stem angiomas. 1): interhemisheric translamina terminalis approach. 2): interhemispheric transcallosal approach. 3): subtemporal transtentorial approach. 4): suboccipital transvermian approach. 5): occipital transtentorial approach.

(1) mixed signal intensity with "reticulated" appearance due to blood clots of different stages, which were more clearly evident than on CT ; $(2)$ on T2 weighted images, a surrounding ring of hypointense signal (a hemosiderin rim); (3) : mild enhancement of the angioma itself using gadopentetate dimeglumin (Gd-DTPA); and $(4)$; clear distinction between the border of the hematoma and the surrounding brain edema.

The surgical approach which we have used thus far are as follows. In case with hypothalamic lesions, we used an interhemispheric translamina terminalis approach or a transcallosal approach. In the dorsal pons and brachium pontis cases, we used a suboccipital transvermian approach. In the case with a lesion between the midbrain and pons, we used a subtemporal transtentorial approach and for the case with a lesion at the superior colliculus, we used an occipital transtentorial approach (Fig. 1). The postoperative course was favorable in all cases and no new deficits following surgery were encountered. Below we present two recent cases which are representative of our experiences.

\section{Representative cases}

Case 8

The patient was a 36 year-old male presenting with Millard-Gublar syndrome at the time of onset. Noncontrast CT revealed a hematoma in the dorsal pons just below the floor of the fourth ventricle. There was slight enhancement of the lesion on contrast CT (Fig 2). 


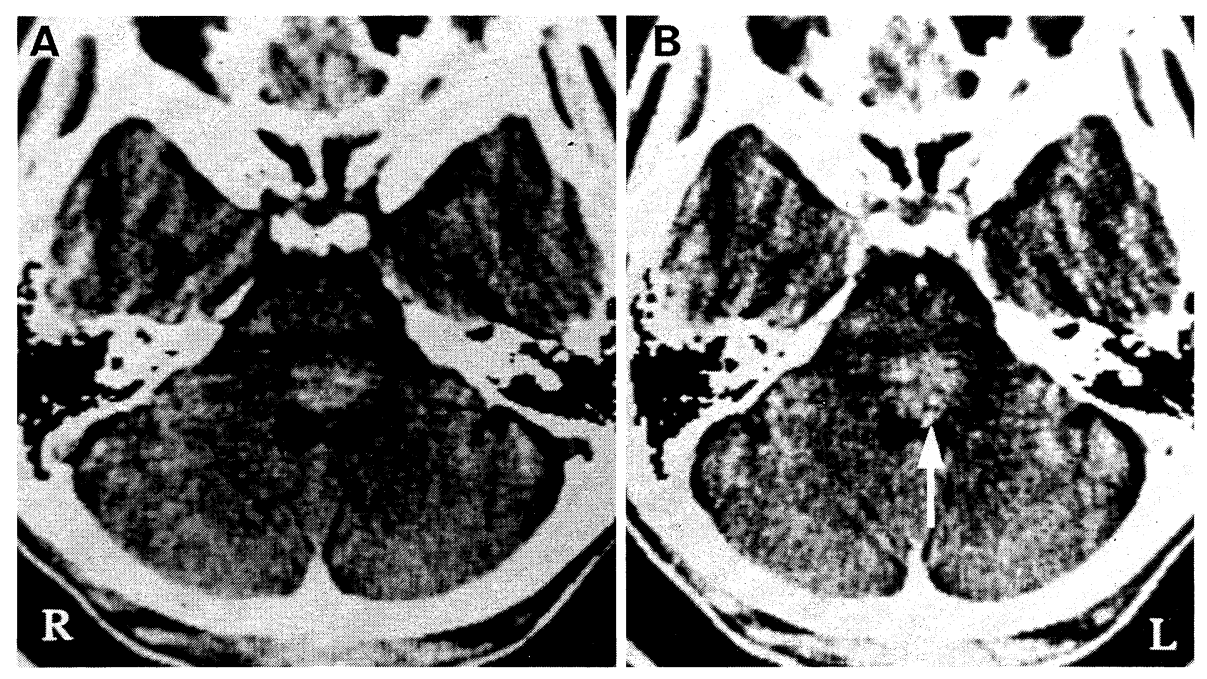

Fig. 2. Case 8. Preoperative CT. Plain CT (A) shows a hematoma in the dorsal part of the pons. Contrast CT (B) shows slight enhancement of the lesion (arrow).

Forty-four after the first attack, he experienced a severe headache and deterioration of the diplopia. CT revealed a second bleeding and he was then admitted to our clinic. Twentyfive days following the rebleeding, surgery was performed through a suboccipital transvermian approach.

After the vermis was split and the fourth ventricle opened, a bulging area of the floor of the fourth ventricle with a deep reddish color was seen. At the surface of this region, a dilated vein running longitudinally was identified. While preserving that vein, a $3 \mathrm{~mm}$ small incision was made in the floor of the ventricle and the old clot was evacuated. The nodule of vascular tissue became visible in the wall of the cavity. It was $5 \mathrm{~mm}$ in diameter with some extremely fine feeding vessels. It was excised. Histopathologically, it was found to be connective tissue containing an sinusoidal vascular network, and was diagnosed as a cavernous angioma. The abnormally dilated vein seen during the operation had been recognized on preoperative angiograms and was thought to be a part of a coexistent venous angioma. Pre- and postoperative MRIs are shown in Fig. 3. Postoperatively, a mild Millard-Gublar syndrome remains, but no new symptoms have appeared and he was discharged able to walk unassisted.

\section{Case 9}

The patient was a 39 year-old male, presenting with a left-sided dysesthesia (tingling sensation). CT taken at the time of onset revealed a hemorrhage from the right superior colliculus to the right thalamus (Fig. 4 A, B). Because symptoms were mild, the patient was managed conservatively, and the hyperdense lesion was found to remain unchanged on follow-up CT studies at regular intervals. After two years, he experienced a sudden headache and upward gaze palsy, and rebleeding was confirmed on CT) (Fig. 4 C, D). On MRI obtained 10 days after the rebleeding, the hematoma showed high signal intensity in T1 weighted images and a mosaic of high and low signal intensities in T2 images (Fig. 5 A, B). The lesion thought to be an angioma itself at the right superior colliculus was found to be slightly enhanced by Gd-DTPA. 


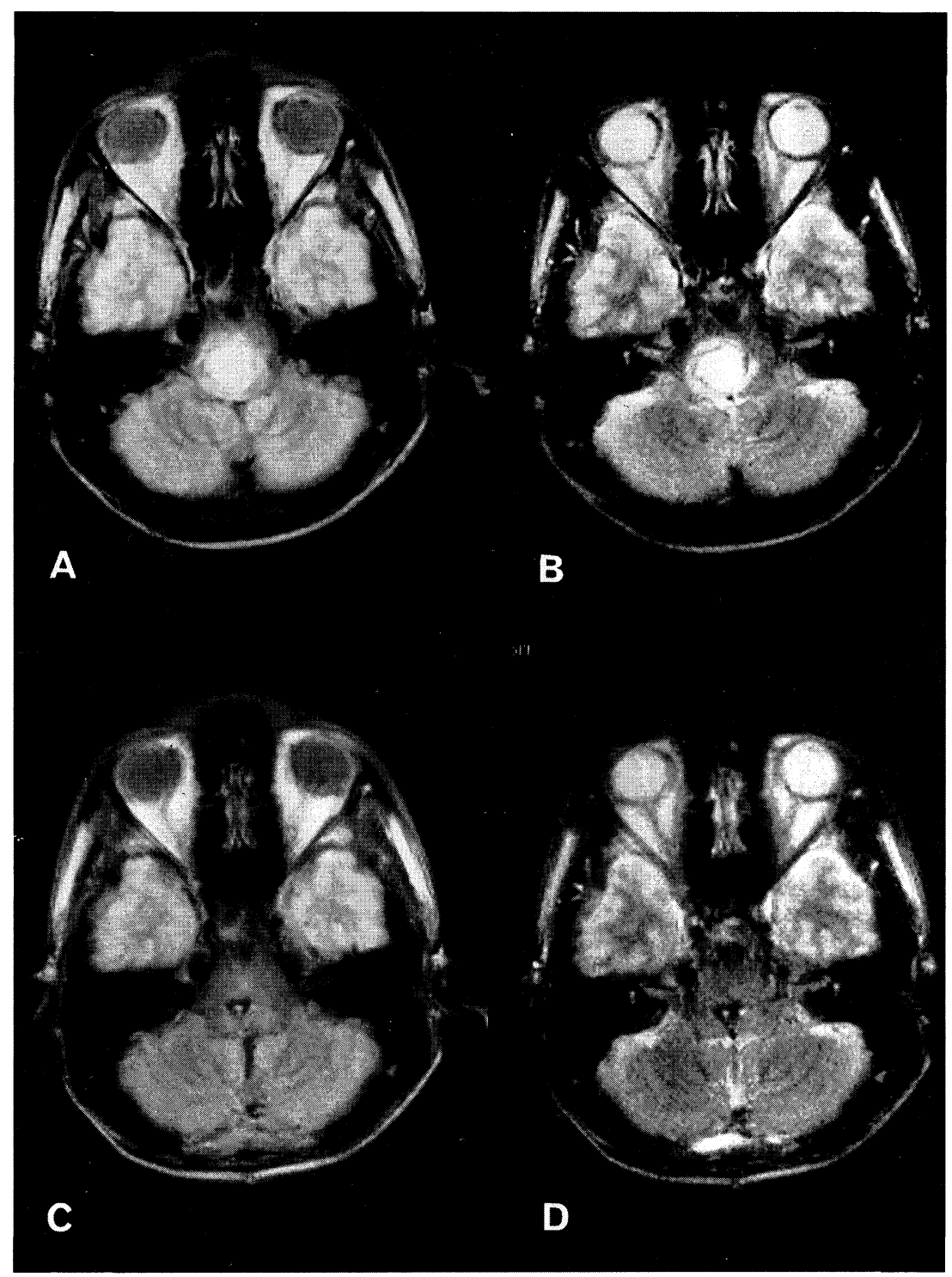

Fig. 3. Case 8. Preoperative $\mathrm{T}_{1}$-weighted (A) and $\mathrm{T}_{2}$-weighted (B) MRIs show homogeneous bright signals, consistent with nonacute hemorrhage. A rim of low signal is noted around the lesion. Postoperative $\mathrm{T}_{1}$-weighted $(\mathrm{C})$ and $\mathrm{T}_{2}$-weighted (D) MRIs show disappearance of the lesion.

One month following the rebleeding, surgery was undertaken through an occipital transtentorial approach with the patient in a prone position. After reaching the free edge of the tentorium, the basal vein of Rosenthal and the vein of Galen in the dense arachnoid membrane were identified once the tentorium had been opened by making an incision parallel to the straight sinus. At the medial side of the right basal vein, the superior colliculus was bulging due to the hematoma and its surface was xanthochromic. Within it, 


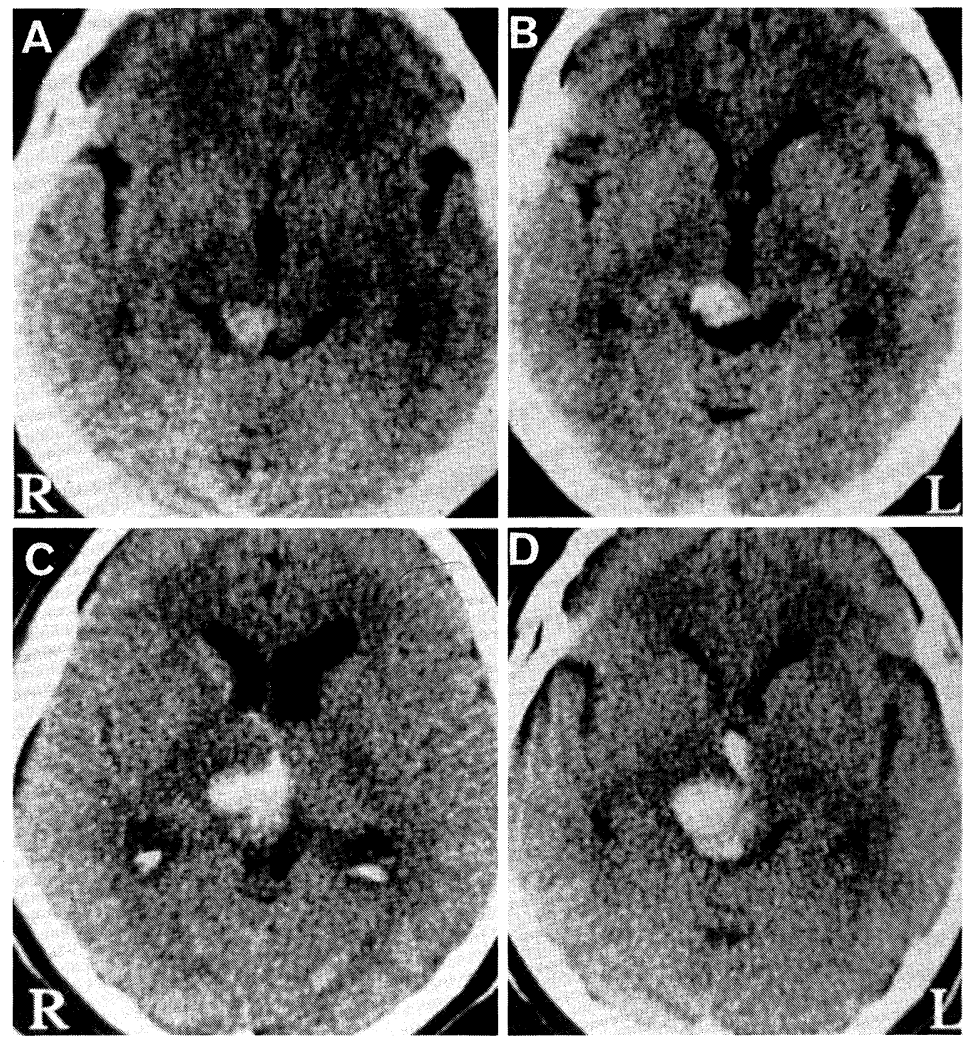

Fig. 4. Case 9. Plain CT $(\mathrm{A}, \mathrm{B})$ at the day of the first bleeding reveals a hematoma in the superior colliculus. Plain CT (C, D) 2 years after the initial onset shows rebleeding at the right superior colliculus and the posterior thalamus with a ventricle hematoma.

however, there was the red-colored angioma-like tissue, which was excised together with aspiration of the liquid hematoma. Auditory brain stem responses (ABR) monitoring during the surgery showed no notable changes. Similar to Case 8, the histopathological study showed a mixture of granulation tissue and thin-walled dilated blood vessels. Postoperative CT and MRI revealed that the entire lesion had been removed (Fig. 5 C, D). Six months after surgery, the upward palsy resolved completely.

\section{Discussion}

Cavernous angiomas can arise from any location in the central nervous system, but frequently arise in the subcortical white matter, basal ganglia and pons. Since the introduction of CT and MRI techniques, the incidence of their detection has greatly increase, particularly those which bleed repeatedly in the brain stem. Many authors have reported the characteristic neuroimaging studies of such lesions (New et al. 1986; Simard et al. 1986; Tagle et al. 1986; 

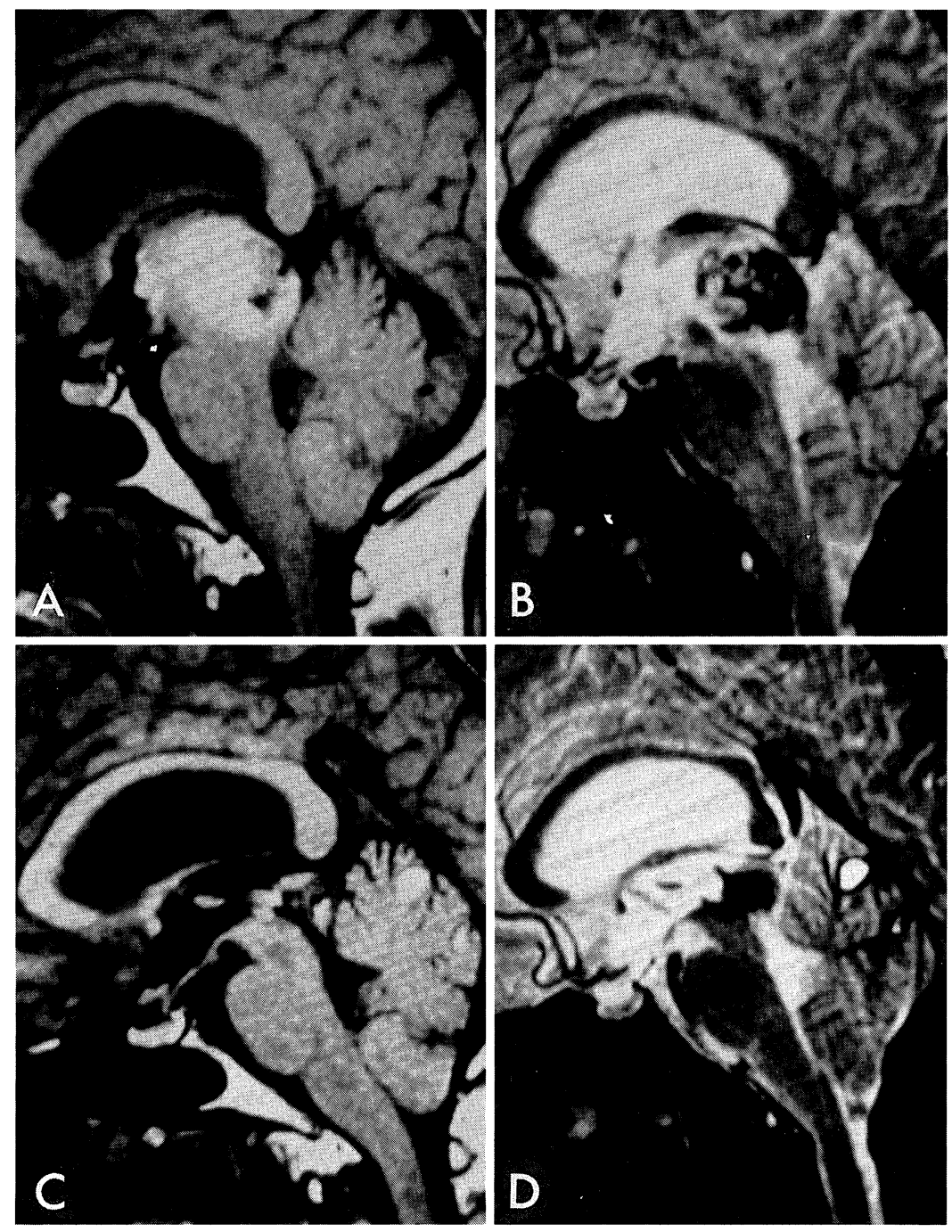

Fig. 5. Case 9. Preoperative $\mathrm{T}_{1}$-weighted (A) and $\mathrm{T}_{2}$-weighted (B) MRIs show a mixed signal intensity lesion within the thalamus and the superior colliculus, consistent with metachronous hemorrhage. Postoperative $\mathrm{T}_{1}$-weighted (C) and $\mathrm{T}_{2}$-weighted (D) MRIs show that the angioma within the superior colliculus was removed via a occipital transtentorial approach with no postoperative deficit.

Rigamonti et al. 1987 ; Labato et al. 1988 ; Ogilvy et al. 1988), but the majority have discussed primarily the findings with regard to the hematoma, rather than with the angioma itself. From the present study, there are two results which deserve emphasis. The first with regard to the CT findings is that a hyperdense area corresponding to the angioma itself remains almost unchanged into the period during which the hyperdensity due a hematoma will have disappeared. Since CT 
number (Hounsfield number) of this prolonged hyperdense area corresponds to that of a hematoma, it is possible to distinguish it from the foci of calcification which are often seen within such lesions. The mechanism underlying this finding is thought to be as follows: Since it is unlikely that repeated small hemorrhages from the angioma would incessantly occur, we believe that the velocity of blood flow within the angioma may be extremely slow, thus making the blood within the vascular spaces to become almost like a clotted blood. The fact that a cavernous angioma is rarely visible in cerebral angiogaphies and the fact that there is little or no enhancement on contrast CT support this supposition. Among our ten cases, we have tried high dose and delayed contrast CT, but we have had no cases of clear-cut enhancement. Contrast CT is, however, effective for delineating venous angiomas which frequently coexist with cavernous angiomas.

A second characteristic finding was found on Gd-DTPA enhanced MRI study of our cases. Although such study has been done in only five cases, all five showed enhancement after Gd-DTPA administration, clearly more distinct that which was seen using contrast CT. We conclude that Gd-DTPA is particularly effective for determining the location of the angioma itself. As has previously been reported concerning hematoma, depending upon the interval since bleeding, the hematoma can show increased or decreased signal intensity and the presence of an lesion of various signal intensities with a reticulated appearance suggests the coexistence of both old and new hematomas. Moreover, on T2 weighted images, an extremely decreased signal intensity ring, the so-called hemosiderin rim, is seen in the periphery of the hematoma (Simard et al. 1986). This shows up most clearly on high-field strength MRI.

Determination of the indications for direct surgery is undoubtedly the most important problem concerning cavernous angiomas of the brain stem. Of course, asymptomatic lesions which are discovered incidentally on CT or MRI study, but which have not bled do not require operative treatment. Since one of the characteristic features of brain stem cavernous angiomas is their recurrent hemorrhage, cases which have bled once might be considered for the direct operation as a means of preventing a second hemorrhage. However, as mentioned above, since the blood flow velocity in cavernous angiomas, unlike most AVMs, is quite slow, it is thought to be extremely rare that a recurrent hemorrhage will lead to a large hematoma. As a rule, we manage the patients with asymptomatic or minimally symptomatic angiomas conservatively with follow-up neuroimaging studies, but perform the direct operation on cases with clearly recurrent hemorrhages or significant neurological deficits. Six of our ten cases have had a second bleeding and fortunately none of the six have had severe sequelae as a result of the rebleeding.

Finally, with regard to the surgical approach, this is determined almost entirely from the location of the angioma (Fig. 1). Only for our case 9, which had the lesion at the superior colliculus, either an occipital transtentorial approach or 
an infratentorial supracerebellar approach could have been used. Both in order to obtain a wider operative exposure and because of the surgeon's previous experiences, the former approach was employed, in retrospect, to have been the appropriate choice. It goes without saying that a particularly careful surgical technique is required for treatment of such deep-seated lesions within the brain stem in order to prevent the appearance of postoperative morbidity, but we have found it unexpectedly easy to excise angiomas in this region without injuring normal brain tissue due to the existence of the hematoma cavity and surrounding gliotic tissue. As a means of assuring an even safer operation, the use of intraoperative evoked potential monitoring ( $\mathrm{ABR}$, etc) is also useful.

\section{References}

1) Konovalov, A.N., Spallone, A., Makhmudov, U.B. \& Ozerova, V.I. (1990) Surgical management of hematomas of the brain stem. J. Neurosurg., 73, 181-186.

2) LeDoux, M.S., Aronin, P.A. \& Odrezin, G.T. (1991) Surgically treated cavernous angiomas of the brain stem: Report of two cases and review of the literature, Surg. Neurol., 35, 395-399.

3) Lobato, R.D., Perez, C., Rivas, J.J. \& Cordobes, R. (1988) Clinical, radiological, and pathological spectrum of angiographically occult intracranial vascular malformations. J. Neurosurg., 68, 518-531.

4) New, P.F.J., Ojemann, R.G., Davis, K.R., Rosen, B.R., Heros, R., Kjellberg, R.N., Adams, R.D. \& Richardson, E.P. (1986) MR and CT of occult vascular malformations of the brain. $A J N R, 7,771-779$.

5) Ogilvy, C.S., Heros, R.C., Ojemann, R.G. \& New, P.F. (1988) Angiographically occult arteriovenous malformations. J. Neurosurg., 69, 350-355.

6) Ondra, S.L., Doty, J.R., Mahla, M.E. \& George, E.D. (1988) Surgical excision of a cavernous hemangioma of the rostral brain stem : Case report. Neurosurgery, 23, 490493.

7) Rigamonti, D., Drayer, B.P., Johnson, P.C., Hadley, M.N., Zabramski, J. \& Spetzler, R.F. (1987) The MRI appearance of cavernous malformations (angiomas). $J$. Neurosurg., 67, 518-524.

8) Simard, J.M., Garcia-Bengochea, F., Ballinger, W.E., Mickle, J.P. \& Quisling, R.G. (1986) Cavernous angioma: A review of 126 collected and 12 new clinical cases. Neurosurgery, 18, 162-172.

9) Tagle, P., Huete, I., Mendez, J. \& Villar, S. (1986) Intracranial cavernous angioma: Presentation and management. J. Neurosurg., 64, 720-723.

10) Tung, H., Giannotta, S.L., Chandrasoma, P.T. \& Zee, C. (1990) Recurrent intraparenchymal hemorrhages from angiographically occult vascular malformations. $J$. Neurosurg., 73, 174-180.

11) Veerapen, R.J., Sbeith, I.A. \& O'Laoire, S.A. (1986) Surgical treatment of cryptic AVM's and associated hematoma in the brain stem and spinal cord. J. Neurosurg., 65, 188-193.

12) Yoshimoto, T. \& Suzuki, J. (1986) Radical surgery on cavernous angioma of the brain stem. Surg. Neurol., 26, 72-78. 